\title{
LINKING THE FK5 TO THE ICRF
}

\author{
F. MIGNARD \& M. FROESCHLE \\ $O C A / C E R G A$ \\ UMR CNRS 6527 \\ Av. Copernic, 06130-Grasse (France)
}

\begin{abstract}
The Hipparcos optical reference frame is compared to the basic FK5 in order to determine the orientation at $T_{0}=1991.25$ and the global spin between the two frames. The components of the spin are significant and suggest a correction the IAU76 value of the precession constant and t.o a possible non-precessional motion of the equinox of the FK5. The regional errors are analysed with harmonic functions and found to be as large as 150 mas in position and 3 mas/yr in proper motion.
\end{abstract}

\section{The FK5 catalogue}

'The FK5 Catalogue has provided until the advent of the ICRF a practical realization of a dynamical frame through the position and proper motion of carefully measured stars. It represents a revision of the FK4 and results from the determination of systematic and individual corrections to the mean positions and proper motions of the FK4, the elimination of the error in the position and motion of the FK4 equinox, and the introduction of the IAU (1976) system of astronomical constants (Fricke et al., 1988). Fricke (1982) has derived the following correction in mas,

$$
E(T)=(525 \pm 45)+(12.75 \pm 1.5)(t-1950.0),
$$

to the sero point of the right ascension. One must bear in mind that any error in the above rate propagates directly into a systematic effect in the system of proper motion and into a defect of inertiality.

The expected positional accuracy of the 1535 bright stars is 30 mas at the mean epoch of the atalogue and $0.6 \mathrm{mas} / \mathrm{yr}$ for the proper motion of the Northern hemisphere and $1.0 \mathrm{mas} / \mathrm{yr}$ for the Southern. By projecting the FK5 positions to the Hipparcos epoch J1991.25, this leads to an expected error in right ascension and declination of 40 to 60 mas, according to the hemisphere.

The 1535 stars of the FK5 have been observed successfully by Hipparcos and their positions are known in 1991.25 with an accuracy of typically $0.4 \pm 0.1 \mathrm{mas}$ in declination and $0.6 \pm 0.2 \mathrm{mas}$ in right ascension, with in the latter case a marked dependence with the declination. The corresponding figures for the proper motions are $0.7 \pm 0.2$ mas $/ y r$ and $0.55 \pm 0.15$ mas $/ y r$.

The Hipparcos Catalogue has been constructed in such a way that the reference frame materialized by the positions coincides with the International Celestial Reference System, within the measurement crrors. The Hipparcos positions and proper motions provide a realisation of an optical non-rotating reference frame with axes pointing to fixed extragalactic directions to within 0.6 mas for the alignment and $0.25 \mathrm{mas} / \mathrm{yr}$ in the rate of rotation, resulting in a similar uncertainty in the intertiality of the system of proper motion (ESA 1997). In terms of consistency, the optical reference frame defined by the Hipparcos positions and proper motions is believed to have a global accuracy of about $0.1 \mathrm{mas}$ and $0.1 \mathrm{mas} / \mathrm{yr}$, with no regional distortion.

\section{The comparison with Hipparcos}

All the multiple stars and objects with questionable solutions in Hipparcos have been excluded from the aralysis and the selection has ended up with 1233 reliable solutions. The reason for excluding 
the double stars and the astrometric binaries, is not in general the quality of the solution, but the fact that the Hipparcos and FK5 astrometric solutions may correspond to two different points on the sky and that the Hipparcos proper motion determined over a timespan shorter than the orbital period does not represent the systematic motion of the barycenter. The comparison is performed at $T_{0}=1991.25$, and for each star one has computed the positional differences $\left(\alpha_{\mathrm{F}}-\alpha_{\mathrm{H}}\right) \cos \delta$ and $\delta_{\mathrm{F}}-\delta_{\mathrm{H}}$ and the proper motion differences, $\left(\mu_{\alpha *}\right)_{\mathrm{F}}-\left(\mu_{\alpha *}\right)_{\mathrm{H}},\left(\mu_{\delta}\right)_{\mathrm{F}}-\left(\mu_{\delta}\right)_{\mathrm{H}}$.

\subsection{GLOBAL ROTATION}

These differences have been projected on a set of orthogonal vectorial harmonics independently for the positional and proper motion differences. The first degree of these harmonics represents the pure rotation, while the harmonics of higher degree account for the zonal differences at decreasing wavelengths with increasing degree. The value of the three angles of the global orientation and of the components of the spin vector are given in Table 1 with their respective uncertainties. The orientation is given at the Hipparcos epoch J1991.25 and at J2000. The standard errors are similar in both cases and there is no need to consider the systematic difference of the order of 0.6 mas between the Hipparcos frame and the ICRS. The sign conventions are such that the rotation represents the orientation of the Hipparcos triad with respect to the FK5 triad or equivalently the three consecutive rotations to align the FK5 triad to the Hipparcos axes.

TABLE 1. Global orientation and spin between the Hipparcos and FK5 Catalogues at J1991.25.

\begin{tabular}{lllllllll}
\hline \multicolumn{3}{c}{ Orientation (mas) } & \multicolumn{4}{c}{ Spin(mas/yr) } \\
& $\mathrm{J} 1991.25$ & $\mathrm{~J} 2000$ & $\sigma$ & & & $\sigma_{\mathrm{H} 1 P}$ & $\sigma_{\mathrm{ICRF}}$ \\
\hline$\epsilon_{x}:$ & -17.3 & -19.9 & 2.3 & $\omega_{x}:$ & -0.30 & 0.10 & 0.27 \\
$\epsilon_{y}:$ & -14.3 & -9.1 & 2.3 & $\omega_{y}:$ & +0.60 & 0.10 & 0.27 \\
$\epsilon_{z}:$ & +22.9 & +16.8 & 2.3 & $\omega_{z}:$ & +0.70 & 0.10 & 0.27 \\
\hline
\end{tabular}

The global rotation has no particular physical meaning, giving the relative orientation of the FK5 frame with respect to the ICRF, insofar as it can be represented by the Hipparcos Catalogue. The three angles of rotation are consistent with the IAU recommendations stipulating that the direction of the Conventional Celestial Pole relative to that of the FK5 should be within 50 mas (Arias et al., 1995). The uncertainty of the orientation with respect to the Hipparcos frame or to the ICRF are similar for the orientation.

The components of the spin are linked to the lack of inertiality of the FK5 frame with respect to the non-rotating extragalactic frame. The uncertainty with respect to Hipparcos is the formal result of the fit, while we have taken into account the uncertainty of the link of $0.25 \mathrm{mas} / \mathrm{yr}$ to determine the true default of inertiality of the FK5 proper motion system. It was combined quadratically, although the difference between the Hipparcos frame and the ICRS should be considered as a systematic bias. Various levels of filtering have been tried to determine this rotation, which proved very stable against the different selections. Comparisons with the bright end of the PPM Catalogue has yielded comparable values for the spin, irrespective of whether the FK5 stars were removed or not. The rotation between the two Catalogues is thus real and can be traced back to the construction of the FK5. We suggest a tentative interpretation based on a correction to the precession constant, $\Delta p$, and a combination of a non-precessional motion of the equinox of the FK5, $\Delta E$, and a correction, $\Delta \lambda$, to the planetary precession, although the latter is likely to be negligible. With,

$$
\begin{aligned}
& \omega_{y}=-\Delta p \sin \epsilon \\
& \omega_{z}=\Delta p \cos \epsilon-(\Delta \lambda+\Delta E)
\end{aligned}
$$

which gives,

$$
\begin{aligned}
\Delta P & =-1.5 \pm 0.7 \mathrm{mas} / \mathrm{yr} \\
\Delta \lambda+\Delta E & =-2.7 \pm 0.7 \mathrm{mas} / \mathrm{yr}
\end{aligned}
$$


The correction to the precession constant is not compatible with the recent determinations based on the VLBI or LLR which are both close to -3 mas/yr. On the other hand, the correction to the non-precessional motion of the equinox is in the range of the uncertainty of \pm 1.5 mas $/$ yr obtained by Fricke.

\subsection{ZONAL DIFFERENCES}

The regional differences are shown in Fig. 1 for the positions at 1991.25 and in Fig. 2 for the components of the proper motion. The magnitude of the systematic differences are much larger than the expected accuracy of the FK5, which strictly speaking applies to small fields. Large systematic differences are conspicuous around the South celestial pole and more generally more significant in the Southern hemisphere than in the Northern. The two plots have a rather similar appearance, indicating that most of the zonal errors in the positions come from the propagation to 1991.25 of the FK5 proper motions. A correction table will be made available and endorsed by the newly appointed Working Group on Reference Systems.

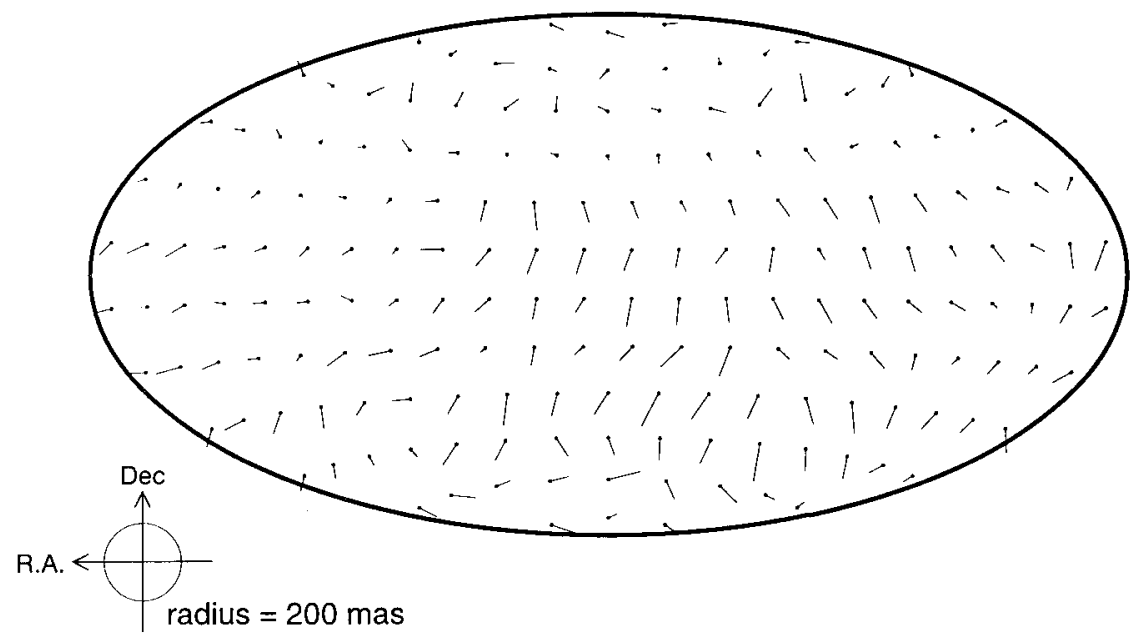

Figure 1. Differences FK5 - HIP for the positions at 1991.25. Right ascension runs right to left from 0 to 360 deg. 


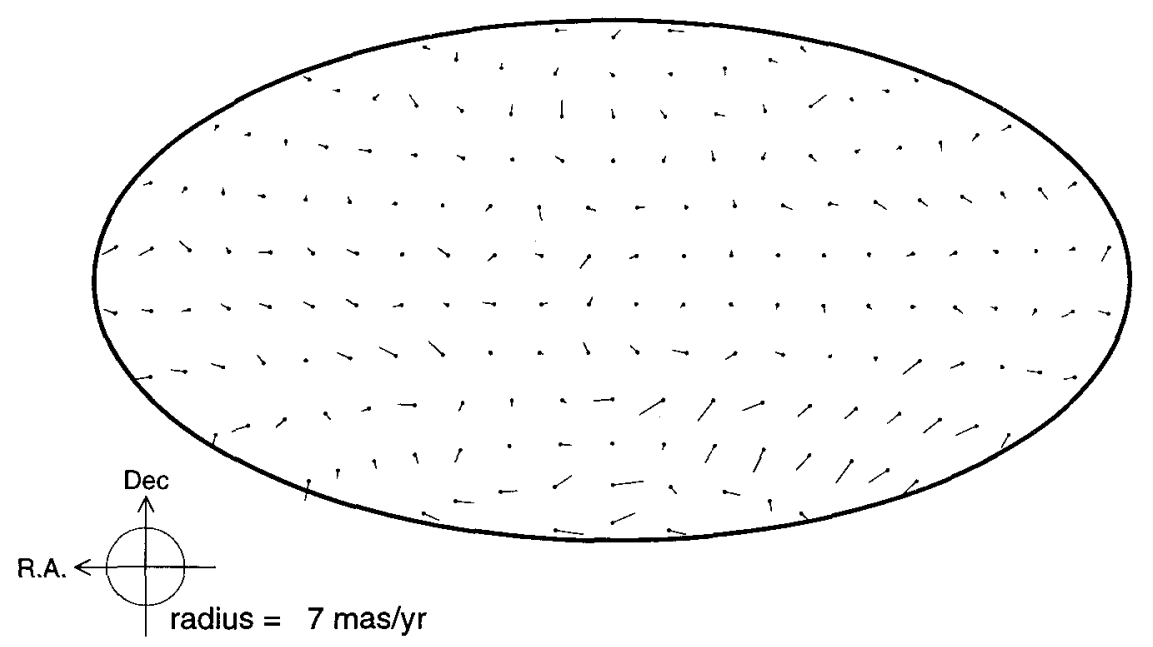

Figure 2. Differences FK5 - HIP for the proper motions. Right ascension runs right to left from 0 to 360 deg.

\section{References}

Arias, E.F., Charlot, P., Feissel, M., Lestrade, J.F., 1995, A\&A, 303, 604-608.

ESA, 1997, The Hipparcos and Tycho Catalogues, SP-1200, Vols. 1-4.

Fricke, W., 1982, A\&A, 107, L13-L16.

Fricke, W., Schwan, H., Lederle, T., 1988, Veröf. Astr. Rechen-Institut, No. 32. 